\title{
Endoplasmic reticulum stress attenuation promotes bovine oocyte maturation in vitro
}

\author{
Hafiza Khatun ${ }^{1,2,3}$, Yasuhiko Wada ${ }^{1,3}$, Toshihiro Konno ${ }^{3,4}$, Hideki Tatemoto ${ }^{3,4}$ and \\ Ken-ichi Yamanaka ${ }^{1,3}$ \\ ${ }^{1}$ Faculty of Agriculture, Saga University, Saga, Japan, ${ }^{2}$ Faculty of Animal Husbandry, Bangladesh Agricultural \\ University, Mymensingh, Bangladesh, ${ }^{3}$ The United Graduate School of Agricultural Sciences, Kagoshima University, \\ Kagoshima, Japan and ${ }^{4}$ Faculty of Agriculture, University of the Ryukyus, Okinawa, Japan \\ Correspondence should be addressed to K Yamanaka; Email: kyama@cc.saga-u.ac.jp
}

\begin{abstract}
We have previously reported that regulation of endoplasmic reticulum (ER) stress during in vitro culture acutely increases bovine embryo developmental rate and cryotolerance; these data indicate that ER stress is a critical factor reducing the quality of in vitro-produced embryos. In the current follow-up study, we examined whether ER stress attenuation during in vitro maturation influences meiotic maturation, oocyte quality, and subsequent embryonic development. Bovine cumulus oocyte complexes (COCs) derived from slaughterhouse ovaries were matured with or without tauroursodeoxycholic acid (TUDCA), a selective inhibitor of ER stress $(0,50,100$, and $200 \mu \mathrm{M})$ for $22 \mathrm{~h}$ followed by in vitro fertilization, and zygotes were cultured for 8 days. Of the different doses of TUDCA, $100 \mu \mathrm{M}$ TUDCA significantly increased the maturation rate, and decreased reactive oxygen species in denuded oocytes, and appeared lower number of apoptotic cells in matured COCs. Subsequently, treatment of TUDCA (100 $\mu \mathrm{M})$ decreased the localization and amount of GRP78/BIP protein level as well as ER stress (GRP78/BIP, PERK, IER1, ATF4, and XBP1) and apoptosis $(C H O P$ and $B A X)$-related gene expression, while it increased the anti-apoptotic gene $B C L 2$ level in matured COCs. Moreover, addition of TUDCA $(100 \mu \mathrm{M})$ during IVM significantly improved the blastocyst formation rate $(43.6 \pm 1.8 \%$ vs $49.7 \pm 1.3 \%)$ and decreased the number of apoptotic cells $(7.7 \pm 1.1 \%$ vs $5.03 \pm 0.6 \%)$ in blastocysts. These findings suggest that the presence of ER stress during maturation impairs the developmental competence of bovine COCs and that this process can be reversed by TUDCA. Reproduction (2020) $159361-370$
\end{abstract}

\section{Introduction}

Mimicking in vivo conditions using in vitro (assisted) reproductive technology is a great challenge for researchers worldwide. Low-efficiency in vitro maturation (IVM) of oocytes has emerged as a crucial issue for poor developmental competence of embryos produced in vitro (Lonergan et al. 2006). Only 30-40\% of oocytes reach the blastocyst stage following IVM, whereas $70 \%$ of in vivo-matured oocytes develop into blastocysts under in vitro conditions (Rizos et al. 2002). The lack of development of these oocytes is probably due to impaired cytoplasmic maturation, which is essential for the ultrastructural and functional modifications of oocytes allowing their fertilization and subsequent embryonic development (Rizos et al. 2002, Reader et al. 2017). Our recent study demonstrated that the use of in vivo-matured oocytes collected from superstimulated Japanese Black Cows enhances the quality of blastocysts produced in vitro compared to the in vitro-matured oocytes (Egashira et al. 2019). Therefore, it is necessary to understand the processes involved in the maturation of the cumulus oocyte complex (COC) in vitro.
Recent evidence suggests that stress to the endoplasmic reticulum (ER) is a major fact for poor developmental competence of oocyte maturation and embryo development in vitro (Lin et al. 2019). Oxidative stress is highly correlated with the function of ER protein-folding pathways; increased cytoplasmic ROS concentrations during maturation alter ER function and trigger ER stress (Sato et al. 2015, Burton et al. 2017). Furthermore, ER stress itself produces ROS and causes mitochondrial dysfunction (Malhotra \& Kaufman 2007). This is important, because mitochondria activity has been linked to embryo development in both oocytes and pre-implanted embryos (Acton et al. 2004, Wu et al. 2010). However, the exact molecular mechanisms of ER stress in mammalian embryo development remain poorly understood, although it is generally accepted that the occurrence of ER stress can be detected by the expression of the unfolded protein response (UPR) pathway mediators, protein kinase-like ER kinase (PERK), activated transcription factor 6 (ATF6), and inositol-requiring enzyme 1 (IRE1) (Tang \& Yang 2015). The 78-kDa glucose-regulated protein (GRP78, also referred to as the immunoglobulin-binding protein 
$(\mathrm{BIP}))$ is an ER chaperone protein that is involved in many cellular processes, including the synthesis and folding new proteins, maintenance of ER calcium homeostasis, and serving as ER stress sensor (Wang et al. 2009). ER stress actively promotes the cell surface localization of GRP78/BIP, resulting in the dissociation of GRP78/BIP from its sensor protein in the ER membrane; furthermore, it activates the cytoprotective UPR (Kadowaki \& Nishitoh 2013, Yang et al. 2016). However, the UPR fails when ER stress is excessive or persistent, and cellular apoptosis is induced through the activation of the CCAAT-enhancer-binding protein homologous protein (CHOP), Jun N-terminal kinase (JNK), and caspase pathway (Rasheva \& Domingos 2009, Namba \& Kodama 2015).

ER stress is involved in ovarian granulosa cell apoptosis (Lin et al. 2012), meiotic maturation (Yang et al. 2012), pre-implantation embryonic development (Yang et al. 2013), and embryo implantation and decidualization (Lan et al. 2013, Lin et al. 2014). Our previous work presents direct evidence that ER stress induction during in vitro culture interferes with the development of bovine embryos, and that this process can be restored by tauroursodeoxycholic acid (TUDCA), an ER stress inhibitor (Khatun et al. 2020). The occurrence of ER stress has also been confirmed during porcine oocyte maturation (Park et al. 2018). Therefore, it has been hypothesized that ER stress during IVM may be contributing to the poor developmental potency of bovine oocytes.

TUDCA is a nontoxic taurine conjugate form of ursodeoxycholic acid (UDCA), a unique bile acid that is mainly found in bears with a trace amount in humans (Nunes et al. 2012). It is a safe cell-protective agent and is approved by the US Food and Drug Administration for the treatment of many diseases such as neurodegenerative disorders, inflammation, osteoarthritis, and diabetes (Vang et al. 2014). Both in vitro and in vivo studies have demonstrated that TUDCA functions as a chemical chaperone; it attenuates ER stress by preventing unfolded protein response dysfunction and stabilizing mitochondria (de Almeida et al. 2007, Chen et al. 2008, Vang et al. 2014). However, to date no investigations have examined the effects of TUDCA on bovine oocyte maturation.

Therefore, the aim of the present study was to determine whether the attenuation of ER stress during in vitro maturation improves the developmental competence of bovine oocytes. First, we investigated the most suitable concentration of TUDCA during IVM by examining the maturation rate, reactive oxygen species in matured denuded oocytes, and apoptosis levels in COCs. Next, to assess the mechanism by which TUDCA counteracts the detrimental effects of ER stress, the localization and amount of the ER stressinducible GRP78/BIP protein expression in matured COCs were detected by immunohistochemistry and western blot analysis, respectively. In addition, realtime quantitative PCR (RT-qPCR) was performed to analyze the expression of genes related to ER stress (GRP78/BIP, PERK, IER1, ATF6, ATF4, XBP1, and CHOP) and apoptosis (CASPASES 3, BAX, and BCL2) in matured COCs. Finally, we explored whether the inhibition of ER stress augments the developmental competence of oocytes by testing the effects of TUDCA supplementation during IVM on subsequent embryonic development in vitro.

\section{Materials and methods}

\section{Chemicals}

All chemicals were purchased from Sigma-Aldrich unless stated otherwise.

\section{Oocyte collection and IVM}

Bovine ovaries were collected from a local abattoir and washed at least three times in sterile saline containing $100 \mathrm{IU} / \mathrm{mL}$ penicillin and $100 \mu \mathrm{g} / \mathrm{mL}$ streptomycin (Seika Pharma, Tokyo, Japan). COCs were aspirated from follicles (2-6 $\mathrm{mm}$ in diameter) using a 19-gauge needle attached to a 10-mL syringe and washed three times with TCM-199 (Thermo Fisher Scientific) containing $5 \%(\mathrm{v} / \mathrm{v})$ fetal bovine serum (FBS, Thermo Fisher Scientific). A group of 50 COCs were matured in a four-well multidish (Nunc, Roskilde, Denmark) containing $500 \mu \mathrm{L}$ TCM-199 supplemented with $5 \% \mathrm{FBS}$, follicle-stimulating hormone $(0.02 \mathrm{IU} / \mathrm{mL}$; Kyoritsu Seiyaku, Tokyo, Japan), and gentamicin $(10 \mu \mathrm{g} / \mathrm{mL}$, Nacalai Tesque, Kyoto, Japan) covered with liquid paraffin (Nacalai Tesque) at $38.5^{\circ} \mathrm{C}$ for $22 \mathrm{~h}$ in a humidified atmosphere of $5 \%$ $\mathrm{CO}_{2}$ in air.

\section{Assessment of meiotic maturation}

After 22 h of IVM, meiotic maturation was assessed by nuclear stages. Oocytes were denuded by pipetting in TCM-199 medium containing $0.1 \%$ hyaluronidase, washed with Dulbecco's PBS (DPBS, Sigma) containing $0.5 \mathrm{mg} / \mathrm{mL}$ polyvinylpyrrolidone (PVP; Nacalai Tesque) and mounted on microscope slides. The samples were fixed for 2-3 days with $25 \%(\mathrm{v} / \mathrm{v})$ acetic acid in ethanol and stained with $1 \%$ acetic orcein ( $\mathrm{w} / \mathrm{v})$ in $45 \%(\mathrm{v} / \mathrm{v})$ acetic acid for $60 \mathrm{~min}$. Meiotic stages of the oocytes were evaluated under a microscope (Eclipse Ti, Nikon).

\section{Culture treatment}

To modulate ER stress, COCs were matured in IVM medium with the ER stress inhibitor TUDCA (sodium salt; Calbiochem). TUDCA was provided as a lyophilized powder, dissolved in distilled water to create $50 \mathrm{mM}$ stock solutions and stored at $4{ }^{\circ} \mathrm{C}$ until further use for up to 2 weeks. Next, the stock solution was diluted in IVM medium to obtain 50-, 100-, and 200- $\mu \mathrm{M}$ TUDCA solutions. 


\section{TUNEL staining}

A TUNEL assay kit (in situ cell death detection kit; Roche) was used to evaluate the presence of apoptotic cells in matured COCs. Briefly, COCs were fixed in $4 \%(\mathrm{w} / \mathrm{v})$ paraformaldehyde solution $(\mathrm{pH} 7.4)$ for $40 \mathrm{~min}$, rinsed three times with $0.05 \%(\mathrm{w} / \mathrm{v})$ PVP-PBS, and then permeabilized with $0.05 \%(\mathrm{w} / \mathrm{v})$ PVP-PBS containing $0.5 \%$ TritonX-100 for $20 \mathrm{~min}$ followed by three washes with $0.05 \%$ (w/v) PVPPBS for $5 \mathrm{~min}$. The fragmented DNA ends of the cells were labeled with fluorescein-dUTP for $60 \mathrm{~min}$ at $38.5^{\circ} \mathrm{C}$. After incubation, COCs were washed three times with $0.05 \%(\mathrm{w} / \mathrm{v})$ PVP-PBS for $5 \mathrm{~min}$ each followed by mounting onto glass slides using mounting solution containing 4',6-diamidino2-phenylindole (DAPI; Vectashield with DAPI; Vector Laboratories, Burlingame, CA, USA). The fluorescence of the fragmented DNA ends was detected under a fluorescence microscope (EVOS ${ }^{\circledR}$ FL, Thermo Fisher Scientific).

\section{Assessment of oxidative stress}

Oxidative stress as assessed by intracellular reactive oxygen species (ROS) in matured denuded oocytes, which were detected by the fluorescent dye 2',7'-dichlorodihydrofluorescein diacetate (DCHFDA; Molecular Probes) as a green fluorescence signal. At least ten denuded oocytes from each treatment group were incubated in PVP-PBS containing $10 \mu \mathrm{M}$ DCHFDA for 15 min at $38.5^{\circ} \mathrm{C}$ in a humidified atmosphere of $5 \% \mathrm{CO}_{2}$ in air followed by two washes with PVP-PBS. The oocytes were then placed on a glass bottom dish, and green fluorescence emission was detected using a fluorescence microscope $\left(\mathrm{EVOS}^{\circledR} \mathrm{FL}\right.$, Thermo Fisher Scientific). The fluorescence intensity of image was quantified using ImageJ software (version 1.55; National Institutes of Health) by counting the number of pixels after color inversion.

\section{$R T-q P C R$ analysis}

RNA extraction and RT-qPCR were carried out according to the procedures described by Khatun et al. (2018). Briefly, total RNA (40 COCs per sample) was isolated using a RNeasy Micro Kit (QIAGEN) according to the manufacturer's instructions. Extracted RNA was either used immediately for RT or stored at $-80^{\circ} \mathrm{C}$. The RT was performed using the SuperScript ${ }^{\mathrm{TM}}$ IV

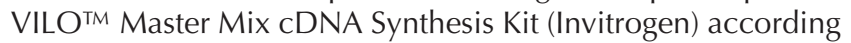
to the manufacturer's instructions. The RT mix $(20 \mu \mathrm{L})$ consisted of $12 \mu \mathrm{L}$ RNA, $4 \mu \mathrm{L}$ SuperScript ${ }^{\text {TM }}$ IV VILO ${ }^{\text {TM }}$ Master Mix, and $4 \mu \mathrm{L}$ nuclease-free water. The RT reaction was performed with annealing of random primers $\left(10 \mathrm{~min}\right.$ at $\left.25^{\circ} \mathrm{C}\right)$, cDNA synthesis $\left(10 \mathrm{~min}\right.$ at $\left.50^{\circ} \mathrm{C}\right)$, and termination of the reaction (5 min at $85^{\circ} \mathrm{C}$ ). Each sample was analyzed in duplicate using the SsoAdvanced ${ }^{\mathrm{TM}}$ universal SYBER ${ }^{\circledR}$ green supermix with CFX Connect (Bio-Rad). The real-time PCR mix $(20 \mu \mathrm{L})$ consisted of $2 \mu \mathrm{L}$ cDNA, $10 \mu \mathrm{L}$ supermix, $6 \mu \mathrm{L}$ nuclease-free water, and $1 \mu \mathrm{L}$ each of forward and reverse primers per gene. The program used for the amplification included a denaturing cycle $(30 \mathrm{~s}$ at $\left.95^{\circ} \mathrm{C}\right), 40$ cycles of PCR $\left(95^{\circ} \mathrm{C}\right.$ for $5 \mathrm{~s}$ and $58^{\circ} \mathrm{C}$ for $\left.5 \mathrm{~s}\right)$, and a melting curve program $\left(60-95^{\circ} \mathrm{C}\right.$ with a heating rate of $0.5^{\circ} \mathrm{C} / \mathrm{s}$, continuous fluorescence acquisition, and cooling to $\left.30^{\circ} \mathrm{C}\right)$. The fold changes of target genes were evaluated by the $\Delta \Delta C T$ method using endogenous reference gene (RN18S1) expression. The PCR primers utilized to amplify each gene are listed in Table 1.

\section{Immunohistochemical detection of GRP78/BIP protein}

After maturation, COCs were washed three times in $0.1 \%$ $(\mathrm{w} / \mathrm{v})$ PVP-PBS and fixed with $4 \%$ paraformaldehyde in PBS for $40 \mathrm{~min}$ at room temperature (RT), followed by three washes with $0.1 \%(\mathrm{w} / \mathrm{v})$ PVP-PBS. COCs were permeabilized with $0.1 \%(\mathrm{w} / \mathrm{v})$ PVP-PBS containing $0.5 \%$ TritonX-100 for $30 \mathrm{~min}$ at RT and blocked in blocking solution $(2 \%(\mathrm{w} / \mathrm{v})$ BSA in $0.1 \%(\mathrm{w} / \mathrm{v})$ PBS-PVA) for $1 \mathrm{~h}$. The samples were incubated with the primary anti-GRP78/BIP (1:200; Cat. No. MA5-27686, Invitrogen) antibody at $4{ }^{\circ} \mathrm{C}$ overnight, washed three times with $0.1 \%(\mathrm{w} / \mathrm{v})$ PVP-PBS containing $0.1 \%(\mathrm{v} / \mathrm{v})$ Tween-20 for $10 \mathrm{~min}$ and then reacted with the anti-mouse fluorescein isothiocyanate (FITC)-conjugated IgG (1:150; Lot No. 064M4788V, Sigma) secondary antibody at RT under dark condition followed by washed three times with $0.1 \%$ $(\mathrm{w} / \mathrm{v})$ PVP-PBS containing $0.1 \%$ (v/v) Tween-20 for $10 \mathrm{~min}$. Primary and secondary antibodies were diluted with Can Get Signal $®$ Immunostain Immunoreaction Enhancer Solution A (TOYOBO, NKB-501). COCs were mounted on slides using mounting medium with DAPI and examined under a confocal laser microscopy (C1, Nikon, Excitation: $488 \mathrm{~nm}$, Emission: $515 \mathrm{~nm}$ ).

\section{Western blot analysis}

Maturated COCs (40 per sample) were washed three times with PBS and lysed in $40 \mu \mathrm{L}$ lysis buffer (Bolt LDS Sample Buffer, Thermo Fisher Scientific) and subsequently denatured at $70^{\circ} \mathrm{C}$ for $10 \mathrm{~min}$. The protein samples were separated by electrophoresis in Bolt ${ }^{\mathrm{TM}} 4-12 \%$ Bis-Tris Plus SDS polyacrylamide precast gels (Invitrogen) and transferred to PVDF membranes using an iBlot@2 Dry Blotting System (Thermo Fisher Scientific). The membranes were blocked by incubation in PVDF-blocking reagent (B1140201; Toyobo, Osaka, Japan) for $1 \mathrm{~h}$ and then washed three times with TBS-T at room temperature. Membranes were incubated with primary anti-GRP78/BIP (1:2000; Cat. No. MA5-27686, Invitrogen, Canada) and anti- $\beta$-actin (1:5000; Cat. No. 01027841, FUJIFILM Wako Pure Chemical Corporation, Osaka, Japan) antibodies at $4{ }^{\circ} \mathrm{C}$ overnight. On the next day, after three washes with TBS-T, membranes were incubated with horseradish peroxidase (HRP)-conjugated goat anti-mouse secondary antibodies (1:30000 dilution; Cat. No. NA931, GE Healthcare) at $37^{\circ} \mathrm{C}$ for $1 \mathrm{~h}$. Primary and secondary antibodies were diluted with immunoreaction enhancer (Can Get Signal; Toyobo). Membranes were washed extensively with TBS-T before bound antibody detection using the ECL-Prime Western Blotting Detection system (GE Healthcare) as per the manufacturer's instructions. Immunoreactive signals were captured and analyzed utilizing NIH image software 1.55 (National Institutes of Health). 
Table 1 Primer information.

\begin{tabular}{lllr}
\hline \multirow{2}{*}{ Gene name } & \multicolumn{2}{c}{ Primer sequence $\left(5^{\prime}-3^{\prime}\right)$} & Product size $($ bp $)$ \\
\cline { 2 - 3 } BAX & Forward & Reverse & 158 \\
CASPASES 3 & CTACTTTGCCAGCAAACTGG & TCCCAAAGTAGGAGAGGA & 169 \\
PERK & CCCAAGTGTGACCACTGAAC & CCATTAGGCCACACTCACTG & 195 \\
GRP78/BIP & GGCTGAAAGATGACAGCACA & AGAACTGGCTCTCGGATGAA & 91 \\
$X B P 1$ & TGCAGCAGGACATCAAGTTC & TTTGTTTGCCCACCTCCAAC & 157 \\
$B C L 2$ & TAGCAGCTCAGACTGCCAGA & ATTCCCCTTGGTCTCTGCTT & 123 \\
ATF6 & TGGATGACCGAGTACCTGAA & GAGACAGCCAGGAGAAATCAAA & 117 \\
ATF4 & TGAACTTCGAGGATGGGTTC & GAATTTGAGCCCTGTTCCAG & 110 \\
CHOP & GGCCAAGCACTTCAAACATC & AAGCATCCTCCTTGCTGTTG & 116 \\
IRE1 & GCACCAAGCATGAACAGTTG & ATCGATGGTGGTTGGGTATG & 108 \\
RN $18 S 1$ & CCGAAGTCAGATGGCATTC & TCTGCAAAGGCTGATGACAG & 138 \\
ACTB & AAACGGCTACCACATCCAAGG & GCGGAAGGATTTAAAGTGGACTC & 405 \\
\hline
\end{tabular}

\section{In vitro embryo production}

In vitro fertilization (IVF) and embryo culture were carried out according to a previously described procedure (Yamanaka et al. 2018). Briefly, frozen semen was thawed by immersing the straw in warm water $\left(37^{\circ} \mathrm{C}\right)$ for $20 \mathrm{~s}$. Spermatozoa were washed by centrifugation ( $800 \mathrm{~g}$ for $10 \mathrm{~min}$ ) in $90 \%(\mathrm{v} / \mathrm{v})$ percoll solution (GE Healthcare). After removing the supernatant, the pellet was diluted with IVF100 solution (Research Institute for the Functional Peptides, Yamagata, Japan) and centrifuged at $600 \mathrm{~g}$ for $5 \mathrm{~min}$. The spermatozoa pellet was then diluted with IVF100 to prepare a final sperm-cell concentration of $5.0 \times 10^{6}$ $\mathrm{sperm} / \mathrm{mL}$. Following IVM, COCs were washed three times with IVF100, and IVF was performed at $38.5^{\circ} \mathrm{C}$ in $5 \% \mathrm{CO}_{2}$ in air under humidified conditions for $6 \mathrm{~h}$ (25 oocytes per $100 \mu \mathrm{L}$ sperm drop covered with liquid paraffin).

After IVF, cumulus cells were removed mechanically by pipetting in CR1 aa medium containing 5\% FBS, and putative zygotes with polar bodies were placed into microdrops (20-25 zygotes per $50 \mu \mathrm{L}$ drop) of CR1 aa medium supplemented with $5 \%(\mathrm{v} / \mathrm{v})$ FBS. The drops were then covered with liquid paraffin and cultured at $38.5^{\circ} \mathrm{C}$ in a humidified atmosphere of $5 \% \mathrm{O}_{2}$ and $5 \% \mathrm{CO}_{2}$ and balanced with $\mathrm{N}_{2}$ through Day 8 (Day 0 represented the day of insemination).

\section{Experimental design}

Experiment 1: Effects of different concentrations of TUDCA on meiotic maturation and ROS content of oocytes

To assess the dose-dependent effects of TUDCA during IVM, bovine COCs were cultured in maturation medium supplemented with different concentrations (0, 50, 100, and $200 \mu \mathrm{M}$ ) of TUDCA at $38.5^{\circ} \mathrm{C}$ for $22 \mathrm{~h}$ in a humidified atmosphere of $5 \% \mathrm{CO}_{2}$ in air and investigated the maturation rate of oocytes by acetic-orcein staining, and the intracellular ROS content by DCHFDA fluorescence.

Experiment 2: Effects of TUDCA on the apoptosis status of $\mathrm{COCS}$

To investigate the effects of TUDCA in reducing ER stress and oocyte quality, COCs were matured with different doses of TUDCA (0, 50, 100, and $200 \mu \mathrm{M})$ and apoptotic cells were detected by using TUNEL assay. Additionally, RT-qPCR analysis was performed to examine apoptosis-associated gene expression in COCs matured with or without TUDCA $(100 \mu \mathrm{M})$.

Experiment 3: Immunohistochemical and western blot analysis of ER stress-induced GRP78/BIP protein in matured COCS

To confirm the presence of ER stress and efficacy of TUDCA against ER stress, the localization and amount of ER chaperone GRP78/BIP protein was detected by both immunohistochemical and western blot analysis in matured COCs obtained from control and TUDCA groups.

\section{Experiment 4: Effects of TUDCA on ER stress-induced genes expression in COCs}

To examine the inhibitory effects of TUDCA on ER stress and its relation to maturation, we analyzed the ER stress-associated genes (those encoding GRP78/BIP, PERK, IER1, ATF4, ATF6, $C H O P$, and $X B P 1)$ expression by RT-qPCR in COCs matured with or without TUDCA $(100 \mu \mathrm{M})$.

Experiment 5: The effects of TUDCA during IVM on subsequent embryonic development in vitro

To assess the effects of ER stress during IVM on subsequent embryonic development, COCs were matured either with or without TUDCA $(100 \mu \mathrm{M})$ and subjected to fertilization followed by embryo culture for 8 days. The developmental competence of embryos was examined by cleavage and blastocyst rates on Days 2 and 8, respectively. The total cell number and apoptotic status of the blastocysts on Day 8 were also determined by using TUNEL assay to investigate embryo quality.

\section{Statistical analysis}

Each trial of experiment run was accompanied by parallel control and same cultural conditions. For each experiment, at least three independent replicates were performed unless specified otherwise. Data are presented as mean \pm S.E.M. All 
statistical analyses were carried out using BellCurve for Excel version 3.20 (Social Survey Research Information, Tokyo, Japan). Differences between two groups were assessed by Student's $t$-test. Differences among three or more groups were examined using ANOVA followed by the Tukey-Kramer multiple comparison test. All percentage data were arcsine transformed prior to statistical analysis. Differences were considered significant at $P<0.05$ and $P<0.01$.

\section{Results}

\section{Effects of TUDCA on the maturation rate and ROS level of oocytes}

The dose-dependent treatment of IVM culture with TUDCA suggested that the $100 \mu \mathrm{M}$ dose added to the maturation medium is effective for ER stress control, since a significantly higher percentage of oocytes progressed to metaphase II (MII) stage in the 100 $\mu M$ TUDCA than in the control group $(89.4 \pm 1.4 \mathrm{vs}$ $79.5 \pm 2.6 ; P<0.01$; Table 2). Consequently, $100 \mu \mathrm{M}$ TUDCA treatment significantly decreased the relative fluorescence intensity of ROS content in comparison with the control group $(P<0.01$; Fig. 1). However, the percentage of MII-stage oocytes (Table 2) and the relative fluorescence intensity of the ROS content (Fig. 1) were similar among the control, 50, and $200 \mu \mathrm{M}$ TUDCA groups. No significant differences were observed in the percentage of GV, GVBD, and metaphase I (MI)-stage oocytes among the groups (Table 2).

\section{Effects of TUDCA on the incidence of apoptosis in COCs}

Based on the TUNEL assay results, the presence of TUNEL-positive cells in the $100 \mu \mathrm{M}$ TUDCA group apparently decreased compared with that in other groups (Fig. 2A). Furthermore, RT-qPCR analysis revealed that treatment of COCs with $100 \mu \mathrm{M}$ TUDCA significantly decreased the pro-apoptotic gene $B A X$ and increased the anti-apoptotic gene $B C L 2$ expression compared with the control group $(P<0.05$; Fig. $2 \mathrm{C}$ and $\mathrm{D})$. However, no significant change was observed for CASPASE 3 expression among the groups.

\section{Effects of TUDCA on GRP78/BIP protein expression in COCs}

As shown in Fig. 3A, both the number of GRP78/BIP protein aggregates and the fluorescence intensity were appeared higher in cumulus cells but not in oocyte under the control group, whereas COCs treated with TUDCA during maturation evidenced lower expression of GRP78/BIP in cumulus cells. In addition, the results of western blot analysis clearly revealed a significant decrease $(P<0.01)$ of GRP78/BIP protein level in COCs treated with TUDCA in comparison with the control group (Fig. 3B and C).

\section{Effects of TUDCA on ER stress-associated genes expression in COCs}

The RT-qPCR analysis clearly revealed that addition of $100 \mu \mathrm{M}$ TUDCA to the maturation medium significantly decreased the expression of ER stress-associated genes (GRP78/BIP, PERK, ATF4, IER1, XBP1, and CHOP) in COCs in comparison with the control group $(P<0.01$; $P<0.05$; Fig. 4). However, TUDCA treatment had no significant effect on ATF6 gene expression.

\section{Effects of TUDCA treatment during IVM on the subsequent embryo development in vitro}

To determine the developmental competence of bovine COCs after inhibition of ER stress during IVM, COCs were subjected to fertilization followed by embryo culture. ER stress during IVM significantly decreased the quality and blastocyst developmental rate, whereas the addition of $100 \mu \mathrm{M}$ TUDCA to the maturation medium significantly improved the blastocyst formation rate $(43.6 \pm 1.8 \%$ vs $49.7 \pm 1.3 \% ; P<0.05$; Fig. $5 \mathrm{~A})$ and decreased the number of TUNEL-positive cells in blastocysts ( $7.7 \pm 1.1 \%$ vs $5.03 \pm 0.6 \%$; $P<0.05$; Fig. $5 \mathrm{D}$ ). However, no significant differences were found in the total cell number and embryo cleavage rate between the groups.

\section{Discussion}

The present study demonstrated that ER stress generally occurs in bovine COCs during IVM and activates the

Table 2 Effects of TUDCA supplementation during in vitro maturation on bovine oocytes.

\begin{tabular}{|c|c|c|c|c|c|}
\hline \multirow[b]{2}{*}{ TUDCA $(\mu \mathrm{M})$} & \multirow[b]{2}{*}{ No. of oocytes examined } & \multicolumn{4}{|c|}{ Meiosis development (\%) } \\
\hline & & GV & GVBD & $\mathrm{MI}$ & MII \\
\hline 0 (Control) & 232 & $1.9 \pm 0.6$ & $4.3 \pm 1.5$ & $14.3 \pm 2.1$ & $79.5 \pm 2.6^{\mathrm{a}}$ \\
\hline 50 & 232 & $1.2 \pm 0.6$ & $4.9 \pm 1.1$ & $14.7 \pm 1.9$ & $81.1 \pm 0.9^{a}$ \\
\hline 100 & 239 & $1.0 \pm 0.5$ & $1.4 \pm 0.7$ & $8.1 \pm 1.1$ & $89.4 \pm 1.4^{b}$ \\
\hline 200 & 236 & $1.8 \pm 0.7$ & $7.1 \pm 2.2$ & $14.2 \pm 2.3$ & $76.9 \pm 3.4^{\mathrm{a}}$ \\
\hline
\end{tabular}

Bovine oocytes were matured with different concentrations of TUDCA for $22 \mathrm{~h}$, and the meiotic maturation rate was examined.

a,b Values with different superscripts within the same column are significantly different $(P<0.01)$.

GV, germinal vesicle; GVBD, germinal vesicle break down; MI, metaphase I; MII, metaphase II; TUDCA, tauroursodeoxycholic acid. Data are mean \pm S.E.M. from seven replicates. 


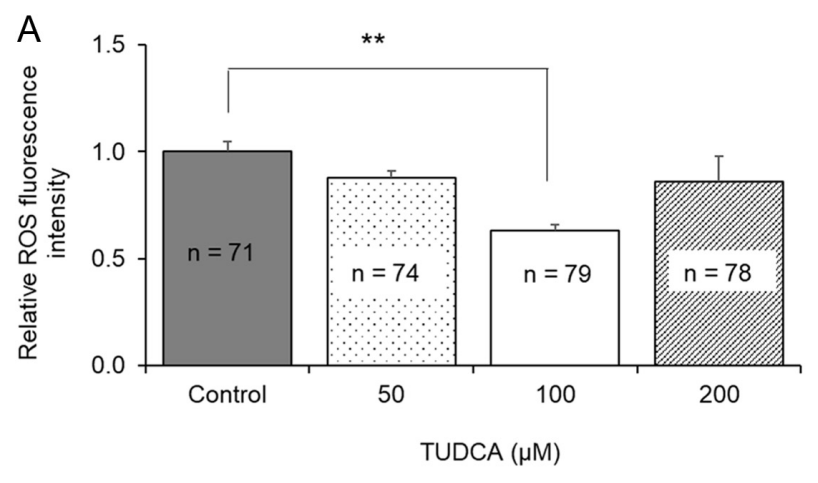

B

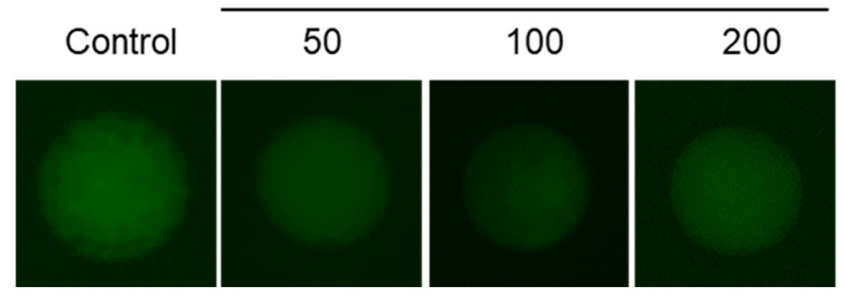

Figure 1 Effects of TUDCA on intracellular ROS generation in oocytes during maturation. (A) Quantification of the relative ROS levels in denuded oocytes from the four different groups. (B) Representative images of ROS fluorescence (green) in denuded oocytes. Scale bar: $400 \mu \mathrm{m}$. Data are means \pm S.E.M. from four replications. ${ }^{* *}$ denotes significant differences $(P<0.01)$. TUDCA, tauroursodeoxycholic acid; ROS, reactive oxygen species.

UPR signaling protein/gene pathways. Furthermore, ER stress inhibition via TUDCA interferes with the effects of ER stress-triggered molecules and retrieves the developmental processes of oocytes. For any chemical treatment, the timing and concentration of the chemical applied is crucial and should be optimized to maximize the output (Kishigami et al. 2006). Different working concentrations of the ER stress inhibitor TUDCA were examined to evaluate their efficiency. We found that $100 \mu \mathrm{M}$ TUDCA significantly increased meiotic maturation and COC quality as evidenced by decreased apoptotic cell number and increased subsequent embryonic development after IVF. To the best of our knowledge, this is the first report to demonstrate that optimized TUDCA concentration is protective in ER stress-induced impairment of bovine oocyte maturation.

The ER is a major intracellular organelle for the synthesis of transmembrane proteins and lipids and is involved in the maintenance of the intracellular calcium homeostasis (Yoshida 2007). A balance between ER protein synthesis and folding machinery is essential for proper protein folding. However, under in vitro conditions many stressors alter the ER function or intracellular homeostasis, leading to the accumulation of misfolded and unfolded proteins, a condition known as ER stress (Kaufman 1999). The literature has disclosed that ER stress and oxidative stress accentuate each other in a positive manner, which increases the ROS production
A
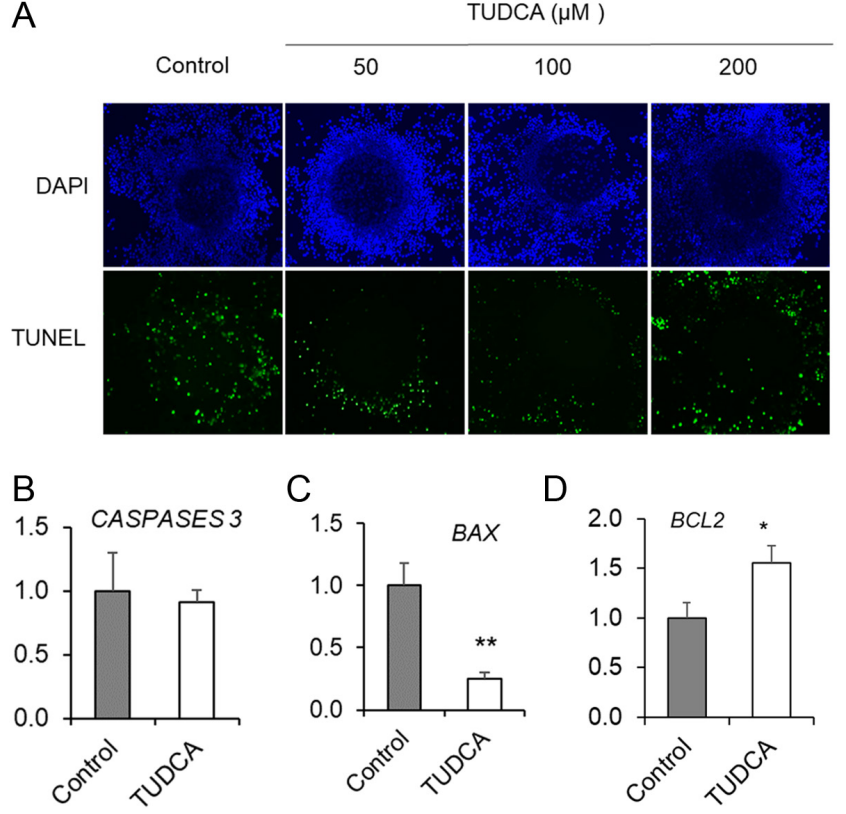

Figure 2 Effects of TUDCA on apoptosis of matured COCs. (A) Representative images of COCs applied for TUNEL staining from four different groups. TUNEL (green) and DAPI (blue) staining were used for apoptotic cells and DNA detection, respectively. Scale bar: 400 $\mu \mathrm{m}$. (B, C and D) The relative expression of CASPASES 3, BAX and BCL2 in COCs obtained from control and $100 \mu \mathrm{M}$ TUDCA group measure by RT-qPCR. Values are means \pm S.E.M. from four independent experiments. ${ }^{*} P<0.05 ; * * P<0.01$. TUDCA, tauroursodeoxycholic acid; COCs, cumulus oocyte complexes; RT-qPCR: real-time quantitative polymerase chain reaction; DAPI, 4',6-diamidino-2-phenylindole; TUNEL, terminal deoxynucleotidyl transferase-mediated dUTP nick-end labeling.

and interferes with mitochondrial function and activates pro-apoptotic signaling pathways (Malhotra \& Kaufman 2007, Yuzefovych et al. 2013, Yoon et al. 2014a). It has been shown that TUDCA can reduce ROS level and modulate mitochondrial turnover (Rodrigues et al. 2003, Fonseca et al. 2017). Taking this fact into consideration, we evaluated the potential of TUDCA to abrogate ROS production and apoptosis. ROS generated by ER stress could affect mitochondria function and finally lead to apoptosis. TUDCA treatment significantly reduced the ROS content, apparently decreased the number of apoptotic cells, significantly downregulated the proapoptotic gene $B A X$ and upregulated the anti-apoptotic gene $B C L 2$ expression in matured COCs. These results indicate that TUDCA mediates cell death and reduces ROS production during maturation of bovine COCs. Moreover, the present study showed that the point at which TUDCA was applied greatly influenced the progress of COCs maturation. During IVM, COCs treated with TUDCA $(100 \mu \mathrm{M})$ significantly decreased the ROS content compared to the control group (Fig. 1), whereas denuded oocytes treated with TUDCA $(100 \mu \mathrm{M})$ showed similar level of ROS production in the control group (Supplementary Fig. 1, see section on 
A
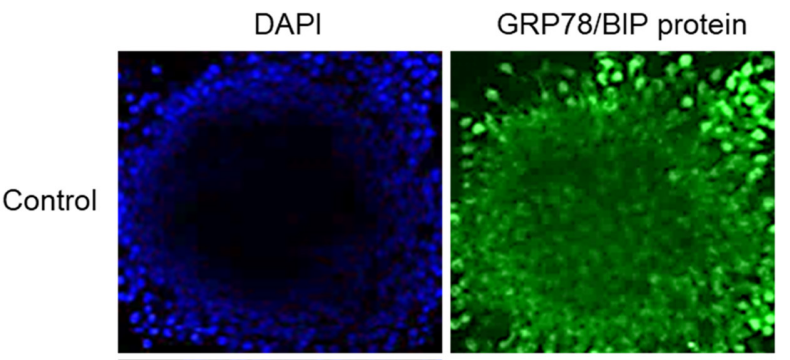

TUDCA
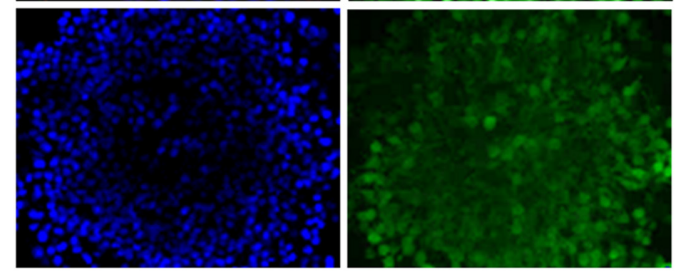

B
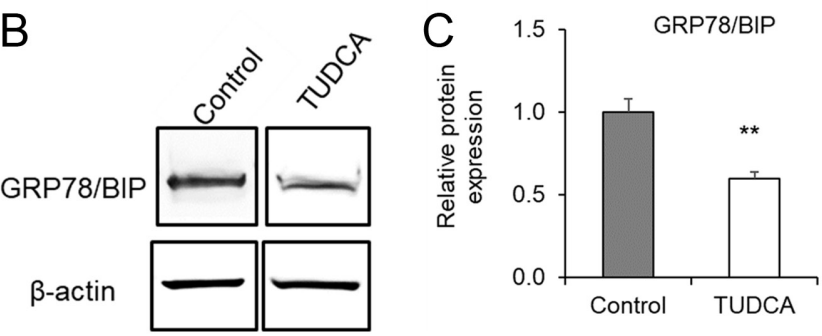

Figure 3 Effects of TUDCA on the localization and amount of GRP78/ BIP protein level in COCs. (A) Immunohistochemical detection of GRP78/BIP protein in matured COCs. (B and C) GRP78/BIP protein and relative expression levels in COCs determined by western blot analysis (five replicates). Values are means \pm S.E.M. and marked with asterisks groups differ significantly $(* * P<0.01)$. COCs, cumulus cell complexes; TUDCA, tauroursodeoxycholic acid.

supplementary materials given at the end of this article), indicating that cumulus cells are more vulnerable to ER stress and diminishes the oocyte quality during maturation. These findings are supported by an elegant work, in which palmitic acids are known to induce

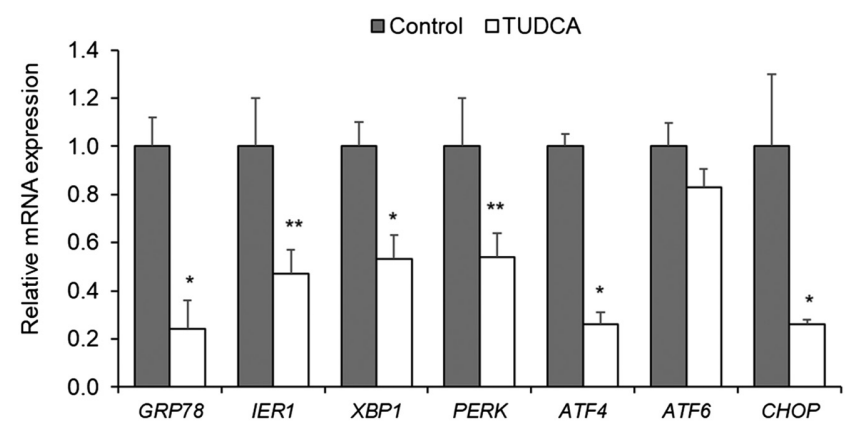

Figure 4 Effects of TUDCA on the relative expression of endoplasmic reticulum (ER) stress-associated genes (GRP78, PERK, ATF4, ATF6, IRE1, XBP1, and CHOP) in COCs measured by RT-qPCR (three replicates) after treated either with or without TUDCA $(100 \mu \mathrm{M})$ during maturation. Values are means \pm S.E.M. and marked with asterisks groups differ significantly $\left({ }^{*} P<0.05 ;{ }^{* *} P<0.01\right)$. COCs, cumulus cell complexes; TUDCA, tauroursodeoxycholic acid; RT-qPCR, real-time quantitative polymerase chain reaction.
A

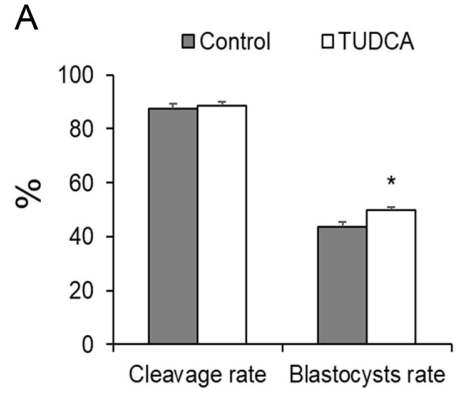

B

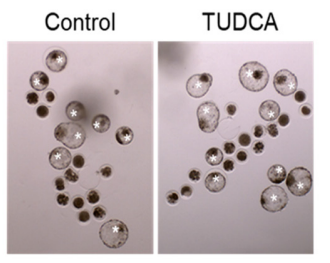

$\mathrm{D}$

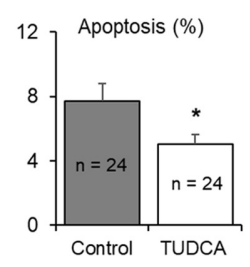

E

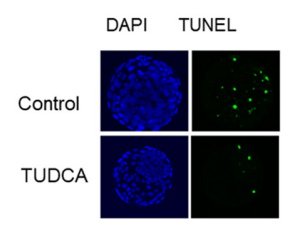

Figure 5 Effects of TUDCA during IVM on the developmental competence of COCs. Bovine COCs were cultured in the presence or absence of TUDCA $(100 \mu \mathrm{M})$ during IVM followed by in vitro fertilization and embryo culture for 8 days. (A) The cleavage and blastocyst developmental rates were examined on Days 2 and 8 , respectively. (B) Representative images of embryos at the blastocyst stage marked by a white star $\left({ }^{*}\right)$. (C) Total cell number and (D) percentage of apoptotic cells in blastocysts. (E) Representative images of Day 8 blastocysts applied for TUNEL staining from the control and TUDCA groups. TUNEL (green) and DAPI (blue) staining were used to detect apoptotic cells and DNA, respectively. The total number of zygotes cultured under control and with TUDCA was 161 and 165 , respectively. The values are means \pm S.E.M. from five independent experiments. ${ }^{*} P<0.05$. Scale bar: $400 \mu \mathrm{m}$. TUDCA: tauroursodeoxycholic acid; IVM: in vitro maturation; COCs, cumulus cell complexes; DAPI: 4',6-diamidino-2-phenylindole; TUNEL: terminal deoxynucleotidyl transferase-mediated dUTP nick-end labeling.

ER stress in mouse COCs during maturation, impair mitochondrial activity and leading to compromise oocyte developmental competence that was reversed by salubrinal, an ER stress inhibitor (Wu et al. 2012).

Furthermore, we also observed that $100 \mu \mathrm{MTUDCA}$ is the optimal concentration for bovine COCs maturation. Maturation rate of oocytes was similar between control and $50 \mu \mathrm{M}$ TUDCA groups and rate was significantly low in comparison with the $100 \mu \mathrm{M}$ TUDCA group; indicating that this concentration was not enough for suppressing ER stress during culture. In contrast, maturation rate tended to decrease and evidently show many apoptotic cells, when TUDCA was administered at a higher $(200 \mu \mathrm{M})$ concentration (Fig. 2A and Table 2). Previous studies have shown that high TUDCA concentrations exhibit toxicity to cultured cells/embryos (Jia et al. 2018, Khatun et al. 2020). This follow-up study also revealed that the optimal concentration of TUDCA for IVM was higher than that of IVC in bovine (Khatun et al. 2020). These findings suggested that the TUDCA 
concentration used for treatment should be optimized depending on the developmental period.

The relief of ER stress by TUDCA treatment is a well characterized, standard in vitro study model. Due to its high antioxidant potential, TUDCA prevents ER calcium depletion (Xie et al. 2002, Boyce et al. 2005), influences Akt-mediated MnSOD activation (Yun et al. 2018), and mitigates ROS generation to directly suppress oxidative stress (Yoon et al. 2014b). Moreover, TUDCA's antiapoptotic properties have been reported by many studies. TUDCA prevents BAX translocation and interrupts the classical apoptosis pathway by modulating mitochondrial membrane perturbation. As a therapeutic agent, TUDCA has been effectively used to improve clinical symptoms and biochemical parameters in several liver diseases without exhibiting major side effects (Lebensztejn 2000, Nousia-Arvanitakis et al. 2001, Vandewynckel et al. 2015). These data supported our observation that TUDCA improves oocyte quality during maturation. In addition, here we speculate that TUDCA enhances the interaction between the oocyte and cumulus cells by reducing the incidence of apoptosis in a manner similar to that described previously (Rodrigues et al. 2003).

In the present study, we investigated the effect of TUDCA on reducing ER stress via the regulation of UPR signaling protein/gene expression in matured COCs. As expected, the localization and amount of GRP78/ BIP protein/mRNA expression significantly decreased in COCs treated with TUDCA, whereas these parameters were significantly increased in control group. In addition, immunohistochemical analysis showed that GPR78/BIP protein appeared higher in cumulus cells but not in oocytes under control group, which is coincided by the ROS level in denuded oocytes after maturation in the present study; clarify that cumulus cells are mainly exposed by ER stress. GRP78/BIP, which is encoded by HSPA5, contributes to protein folding in the ER compartment, and higher expression of GRP78/BIP at the transcriptional level is a marker of cellular ER stress (Park et al. 2017). The activation of GRP78/BIP regulates the PERK-elF2a pathway, which induces the transcription factors ATF4 and CHOP, markers of apoptosis initiation (Wang et al. 2013). A recent study reported that the UPR signaling arm PERK-ATF4 influences the expression of $I R E 1 \alpha$, thereby elevating the ratio of XBP1 mRNA splicing (Tsuru et al. 2016). Taking these data into consideration, we reasoned that the increase in the ER chaperone GRP78/BIP expression at the protein or mRNA level activates the UPR signaling genes PERK, ATF4, CHOP, $I E R 1$, and XBP1 in COCs during maturation. Importantly, TUDCA treatment significantly decreased the expression of these genes, indicating that the ER stress-induced UPR signaling arm PERK-eIF2a-ATF4-CHOP or IER1-XBP1 is inhibited by TUDCA. These findings were also supported by the downregulation of the pro-apoptotic gene $B A X$ in COCs treated with TUDCA, because this apoptotic molecule interacted directly with the cytosolic domain of IRE1 during excessive ER stress (Hetz et al. 2006).
However, we did not detect any significant effect of TUDCA on ATF6 gene expression, suggesting that protein translation was less affected. These results are supported by many studies implicating TUDCA as a chemical chaperone that can mitigate ER stress-induced UPR signaling (Uppala et al. 2017).

Finally, we demonstrated that inhibition of ER stress via TUDCA during IVM significantly $(P<0.05)$ improves the developmental rate and quality of embryos after IVF. Similar observation was found in porcine, where TUDCA promotes maturation of COCs and embryonic development by inhibiting ER stress (Zhang et al. 2012). However, the remedial mechanism through which TUDCA exerts its effect on the maturation of oocytes and improves their further embryonic development is not entirely clear yet. We speculate here that the beneficial effect of TUDCA on embryo development after IVF may rely upon the oocyte quality obtained during IVM, which probably maintains the redox homeostasis and prevents oxidative stress after IVM. We also used salubrinal as another ER stress inhibitor and found that salubrinal $(400 \mathrm{nM})$ supplementation in IVM has no significant effect on the rate of oocyte maturation or subsequent embryonic development (Supplementary Table 1), which is similar to result reported by a previous study showing that treatment of salubrinal during IVM has no significant effect on oocyte maturation or embryonic development under normal conditions, although it attenuates nonesterified fatty acids (NEFAs) induced-ER stress in bovine COCs as indicated by increased subsequent embryonic development in vitro (Sutton-McDowall et al. 2016). Collectively, these results confirm that salubrinal is not as effective as TUDCA in the regulation of ER stress during maturation of bovine COCs. Interestingly, a comparative study demonstrated by Park et al. (2019) clearly show that the efficacy of TUDCA against ER stress is higher than salubrinal due to their different structure and mode of action. Salubrinal appears to be cytoprotective during ER stress by maintaining eukaryotic translation initiation factor 2 alpha (elF2 $\alpha$ ) phosphorylation and to reduce global protein synthesis (Boyce et al. 2005). Therefore, salubrinal is an elF $2 \alpha$-specific ER stress inhibitor. Whereas TUDCA is a hydrophilic bile acid and acts as bile secretagogues and immunomodulators that can stabilize the ER stress-induced UPR signaling pathway (Zhang et al. 2012, Vandewynckel et al. 2015). This high protective property of TUDCA may also lie in the presence of a taurine group, which enhances glucose metabolism by stimulating mitochondrial metabolic functions (Nunes et al. 2012). In the literature, TUDCA has been widely reported as a strong ER stress inhibitor and to improve the quality and developmental competence of oocytes/ embryos (Song et al. 2012, Basar et al. 2014, Yoon et al. 2014b, Khatun et al. 2020).

In conclusion, our results show that ER stress during IVM is a crucial fact for the poor developmental competence of bovine COCs. Whereas treatment of COCs with ER stress inhibitor TUDCA during IVM 
improves the bovine oocyte maturation and subsequent embryonic development by suppressing ER stress via regulation of UPR signaling pathways, ROS generation and apoptosis in COCs during maturation.

\section{Supplementary materials}

This is linked to the online version of the paper at https://doi. org/10.1530/REP-19-0492.

\section{Declaration of interest}

The authors declare that there is no conflict of interest that could be perceived as prejudicing the impartiality of the research reported.

\section{Funding}

This study was supported in part by a grant awarded to $\mathrm{K} \mathrm{Y}$ by the Japan Society for the Promotion of Science (KAKENHI Grant No. JP17K15361).

\section{Author contribution statement}

$\mathrm{H} \mathrm{K}$ designed and performed the experiments, analyzed data, and wrote the manuscript; Y W, T K and H T participated in the conception of the study design and revised the manuscript; $K$ $\mathrm{Y}$ conceived the study design, participated in data analysis and interpretation, and revised the manuscript.

\section{Acknowledgments}

The authors thank Sagaken-Chikusan-Kousha for gifting them the bovine ovaries.

\section{References}

Acton BM, Jurisicova A, Jurisica I \& Casper RF 2004 Alterations in mitochondrial membrane potential during preimplantation stages of mouse and human embryo development. Molecular Human Reproduction 10 23-32. (https://doi.org/10.1093/molehr/gah004)

Basar M, Bozkurt I, Guzeloglu-Kayisli O, Sozen B, Tekmen I, Schatz F, Arici A, Lockwood CJ \& Kayisli UA 2014 Unfolded protein response prevents blastocyst formation during preimplantation embryo development in vitro. Fertility and Sterility 102 1777-1784. (https://doi. org/10.1016/j.fertnstert.2014.09.004)

Boyce M, Bryant KF, Jousse C, Long K, Harding HP, Scheuner D, Kaufman RJ, Ma D, Coen DM, Ron D et al. 2005 A selective inhibitor of elF2alpha dephosphorylation protects cells from ER stress. Science $\mathbf{3 0 7}$ 935-939. (https://doi.org/10.1126/science.1101902)

Burton GJ, Yung HW \& Murray AJ 2017 Mitochondrial - endoplasmic reticulum interactions in the trophoblast: stress and senescence. Placenta 52 146-155. (https://doi.org/10.1016/j.placenta.2016.04.001)

Chen Y, Liu CP, Xu KF, Mao XD, Lu YB, Fang L, Yang JW \& Liu C 2008 Effect of taurine-conjugated ursodeoxycholic acid on endoplasmic reticulum stress and apoptosis induced by advanced glycation end products in cultured mouse podocytes. American Journal of Nephrology 28 1014-1022. (https://doi.org/10.1159/000148209)

de Almeida SF, Picarote G, Fleming JV, Carmo-Fonseca M, Azevedo JE \& de Sousa M 2007 Chemical chaperones reduce endoplasmic reticulum stress and prevent mutant HFE aggregate formation. Journal of Biological Chemistry 282 27905-27912. (https://doi.org/10.1074/jbc. M702672200)
Egashira J, Ihara Y, Khatun H, Wada Y, Konno T, Tatemoto H \& Yamanaka KI 2019 Efficient in vitro embryo production using in vivo-matured oocytes from superstimulated Japanese Black cows. Journal of Reproduction and Development 65 183-190. (https://doi.org/10.1262/jrd.2018-155)

Fonseca I, Gordino G, Moreira S, Nunes MJ,Azevedo C, GamaMJ, Rodrigues E, Rodrigues CMP \& Castro-Caldas M 2017 Tauroursodeoxycholic acid protects against mitochondrial dysfunction and cell death via mitophagy in human neuroblastoma cells. Molecular Neurobiology 54 6107-6119. (https://doi.org/10.1007/s12035-016-0145-3)

Hetz C, Bernasconi P, Fisher J, Lee AH, Bassik MC, Antonsson B, Brandt GS, Iwakoshi NN, Schinzel A, Glimcher LH et al. 2006 Proapoptotic BAX and BAK modulate the unfolded protein response by a direct interaction with IRE1alpha. Science 312 572-576. (https://doi. org/10.1126/science.1123480)

Jia Z, He Q, Shan C \& Li F 2018 Tauroursodeoxycholic acid attenuates gentamicin-induced cochlear hair cell death in vitro. Toxicology Letters 294 20-26. (https://doi.org/10.1016/j.toxlet.2018.05.007)

Kadowaki H \& Nishitoh H 2013 Signaling pathways from the endoplasmic reticulum and their roles in disease. Genes 4 306-333. (https://doi. org/10.3390/genes4030306)

Kaufman RJ 1999 Stress signaling from the lumen of the endoplasmic reticulum: coordination of gene transcriptional and translational controls. Genes and Development 13 1211-1233. (https://doi.org/10.1101/ gad.13.10.1211)

Khatun H, Egashira J, Sakatani M, Takenouchi N, Tatemoto H, Wada Y \& Yamanaka KI 2018 Sericin enhances the developmental competence of heat-stressed bovine embryos. Molecular Reproduction and Development 85 696-708. (https://doi.org/10.1002/mrd.23038)

Khatun H, Ihara Y, Takakura K, Egashira J, Wada Y, Konno T, Tatemoto H \& Yamanaka KI 2020 Role of endoplasmic reticulum stress on developmental competency and cryo-tolerance in bovine embryos. Theriogenology 142 131-137. (https://doi.org/10.1016/j.theriogenology.2019.09.042)

Kishigami S, Mizutani E, Ohta H, Hikichi T, Thuan NV, Wakayama S, Bui HT \& Wakayama T 2006 Significant improvement of mouse cloning technique by treatment with trichostatin A after somatic nuclear transfer. Biochemical and Biophysical Research Communications 340 183-189. (https://doi.org/10.1016/j.bbrc.2005.11.164)

Lan X, Jin Y, Yang Y, Lin P, Hu L, Cui C, Li Q, Li X \& Wang A 2013 Expression and localization of Luman RNA and protein during mouse implantation and decidualization. Theriogenology 80 138-144.e1-2. (https://doi. org/10.1016/j.theriogenology.2013.02.024)

Lebensztejn DM 2000 Application of ursodeoxycholic acid (UDCA) in the therapy of liver and biliary duct diseases in children. Medical Science Monitor 6 632-636.

Lin P, Yang Y, Li X, Chen F, Cui C, Hu L, Li Q, Liu W \& Jin Y 2012 Endoplasmic reticulum stress is involved in granulosa cell apoptosis during follicular atresia in goat ovaries. Molecular Reproduction and Development 79 423-432. (https://doi.org/10.1002/mrd.22045)

Lin P, Jin Y, Lan X, Yang Y, Chen F, Wang N, Li X, Sun Y \& Wang A 2014 GRP78 expression and regulation in the mouse uterus during embryo implantation. Journal of Molecular Histology 45 259-268. (https://doi. org/10.1007/s10735-013-9552-1)

Lin CC, Hsiao LD, Cho RL \& Yang CM 2019 Carbon monoxide releasing molecule-2-upregulated ROS-dependent heme oxygenase-1 axis suppresses lipopolysaccharide-induced airway inflammation. International Journal of Molecular Sciences 20. (https://doi.org/10.3390/ ijms20133157)

Lonergan P, Fair T, Corcoran D \& Evans AC 2006 Effect of culture environment on gene expression and developmental characteristics in IVF-derived embryos. Theriogenology 65 137-152. (https://doi. org/10.1016/j.theriogenology.2005.09.028)

Malhotra JD \& Kaufman RJ 2007 Endoplasmic reticulum stress and oxidative stress: a vicious cycle or a double-edged sword? Antioxidants and Redox Signaling 9 2277-2293. (https://doi.org/10.1089/ars.2007.1782)

Namba T \& Kodama R 2015 Avarol induces apoptosis in pancreatic ductal adenocarcinoma cells by activating PERK-elF2alpha-CHOP signaling. Marine Drugs 13 2376-2389. (https://doi.org/10.3390/md13042376)

Nousia-Arvanitakis S, Fotoulaki M, Economou H, Xefteri M \& GalliTsinopoulou A 2001 Long-term prospective study of the effect of ursodeoxycholic acid on cystic fibrosis-related liver disease. Journal of Clinical Gastroenterology 32 324-328. (https://doi. org/10.1097/00004836-200104000-00009) 
Nunes AF, Amaral JD, Lo AC, Fonseca MB, Viana RJ, Callaerts-Vegh Z, D'Hooge R \& Rodrigues CM 2012 TUDCA, a bile acid, attenuates amyloid precursor protein processing and amyloid-beta deposition in APP/PS1 mice. Molecular Neurobiology 45 440-454. (https://doi. org/10.1007/s12035-012-8256-y)

Park HJ, Park JY, Kim JW, Yang SG, Jung JM, Kim MJ, Park JJ \& Koo DB 2017 Regulation of the endoplasmic reticulum Stress by BIP/GRP78 is involved in meiotic maturation of porcine oocytes in vitro. Development and Reproduction 21 407-415. (https://doi.org/10.12717/DR.2017.21.4.407)

Park HJ, Park JY, Kim JW, Yang SG, Jung JM, Kim MJ, Kang MJ, Cho YH, Wee G, Yang HY et al. 2018 Melatonin improves the meiotic maturation of porcine oocytes by reducing endoplasmic reticulum stress during in vitro maturation. Journal of Pineal Research 64 e12458. (https://doi. org/10.1111/jpi.12458)

Park YR, Park HB, Kim MJ, Jung BD, Lee S, Park CK \& Cheong HT 2019 Effects of endoplasmic reticulum stress inhibitor treatment during the micromanipulation of somatic cell nuclear transfer in porcine oocytes. Development and Reproduction 23 43-54. (https://doi.org/10.12717/ DR.2019.23.1.043)

Rasheva VI \& Domingos PM 2009 Cellular responses to endoplasmic reticulum stress and apoptosis. Apoptosis 14 996-1007. (https://doi. org/10.1007/s10495-009-0341-y)

Reader KL, Stanton JL \& Juengel JL 2017 The role of oocyte organelles in determining developmental competence. Biology 6 35. (https://doi. org/10.3390/biology6030035)

Rizos D, Ward F, Duffy P, Boland MP \& Lonergan P 2002 Consequences of bovine oocyte maturation, fertilization or early embryo development in vitro versus in vivo: implications for blastocyst yield and blastocyst quality. Molecular Reproduction and Development 61 234-248. (https:// doi.org/10.1002/mrd.1153)

Rodrigues CM, Sola S, Sharpe JC, Moura JJ \& Steer CJ 2003 Tauroursodeoxycholic acid prevents Bax-induced membrane perturbation and cytochrome $\mathrm{C}$ release in isolated mitochondria. Biochemistry 42 3070-3080. (https://doi.org/10.1021/bi026979d)

Sato AY, Tu X, McAndrews KA, Plotkin LI \& Bellido T 2015 Prevention of glucocorticoid induced-apoptosis of osteoblasts and osteocytes by protecting against endoplasmic reticulum (ER) stress in vitro and in vivo in female mice. Bone 73 60-68. (https://doi.org/10.1016/j.bone.2014.12.012)

Song BS, Yoon SB, Kim JS, Sim BW, Kim YH, Cha JJ, Choi SA, Min HK, Lee Y, Huh JW et al. 2012 Induction of autophagy promotes preattachment development of bovine embryos by reducing endoplasmic reticulum stress. Biology of Reproduction 87 8, 1-11. (https://doi.org/10.1095/ biolreprod.111.097949)

Sutton-McDowall ML, Wu LL, Purdey M, Abell AD, Goldys EM, MacMillan KL, Thompson JG \& Robker RL 2016 Nonesterified fatty acidinduced endoplasmic reticulum stress in cattle cumulus oocyte complexes alters cell metabolism and developmental competence. Biology of Reproduction 94 23. (https://doi.org/10.1095/biolreprod.115.131862)

Tang HZ \& Yang LM 2015 Activation of the unfolded protein response in aged human lenses. Molecular Medicine Reports 12 389-393. (https:// doi.org/10.3892/mmr.2015.3417)

Tsuru A, Imai Y, Saito M \& Kohno K 2016 Novel mechanism of enhancing IRE1alpha-XBP1 signalling via the PERK-ATF4 pathway. Scientific Reports 6 24217. (https://doi.org/10.1038/srep24217)

Uppala JK, Gani AR \& Ramaiah KVA 2017 Chemical chaperone, TUDCA unlike PBA, mitigates protein aggregation efficiently and resists ER and non-ER stress induced HepG2 cell death. Scientific Reports 73831. (https://doi.org/10.1038/s41598-017-03940-1)

Vandewynckel YP, Laukens D, Devisscher L, Paridaens A, Bogaerts E, Verhelst X, Van den Bussche A, Raevens S, Van Steenkiste C, Van Troys M et al. 2015 Tauroursodeoxycholic acid dampens oncogenic apoptosis induced by endoplasmic reticulum stress during hepatocarcinogen exposure. Oncotarget 6 28011-28025. (https://doi.org/10.18632/oncotarget.4377)

Vang S, Longley K, Steer CJ \& Low WC 2014 The unexpected uses of urso- and tauroursodeoxycholic acid in the treatment of non-liver diseases. Global Advances in Health and Medicine 3 58-69. (https://doi. org/10.7453/gahmj.2014.017)

Wang M, Wey S, Zhang Y, Ye R \& Lee AS 2009 Role of the unfolded protein response regulator GRP78/BiP in development, cancer, and neurological disorders. Antioxidants and Redox Signaling 11 2307-2316. (https://doi. org/10.1089/ars.2009.2485)
Wang F, Tian X, Zhang L, Tan D, Reiter RJ \& Liu G 2013 Melatonin promotes the in vitro development of pronuclear embryos and increases the efficiency of blastocyst implantation in murine. Journal of Pineal Research 55 267-274. (https://doi.org/10.1111/jpi.12069)

Wu LL, Dunning KR, Yang X, Russell DL, Lane M, Norman RJ \& Robker RL 2010 High-fat diet causes lipotoxicity responses in cumulus-oocyte complexes and decreased fertilization rates. Endocrinology 151 5438-5445. (https://doi.org/10.1210/en.2010-0551)

Wu LL, Russell DL, Norman RJ \& Robker RL 2012 Endoplasmic reticulum (ER) stress in cumulus-oocyte complexes impairs pentraxin-3 secretion, mitochondrial membrane potential (DeltaPsi m), and embryo development. Molecular Endocrinology 26 562-573. (https://doi. org/10.1210/me.2011-1362)

Xie Q, Khaoustov VI, Chung CC, Sohn J, Krishnan B, Lewis DE \& Yoffe B 2002 Effect of tauroursodeoxycholic acid on endoplasmic reticulum stress-induced caspase-12 activation. Hepatology 36 592-601. (https:// doi.org/10.1053/jhep.2002.35441)

Yamanaka KI, Khatun H, Egashira J, Balboula AZ, Tatemoto H, Sakatani M, Takenouchi N, Wada Y \& Takahashi M 2018 Heat-shockinduced cathepsin B activity during IVF and culture compromises the developmental competence of bovine embryos. Theriogenology 114 293-300. (https://doi.org/10.1016/j.theriogenology.2018.04.005)

Yang X, Wu LL, Chura LR, Liang X, Lane M, Norman RJ \& Robker RL 2012 Exposure to lipid-rich follicular fluid is associated with endoplasmic reticulum stress and impaired oocyte maturation in cumulusoocyte complexes. Fertility and Sterility 97 1438-1443. (https://doi. org/10.1016/j.fertnstert.2012.02.034)

Yang $Y$, Jin $Y$, Martyn AC, Lin P, Song Y, Chen F, Hu L, Cui C, Li X, Li Q et al. 2013 Expression pattern implicates a potential role for luman recruitment factor in the process of implantation in uteri and development of preimplantation embryos in mice. Journal of Reproduction and Development 59 245-251. (https://doi. $\operatorname{org} / 10.1262 / j r d .2012-137)$

Yang Y, Pei X, Jin Y, Wang Y \& Zhang C 2016 The roles of endoplasmic reticulum stress response in female mammalian reproduction. Cell and Tissue Research 363 589-597. (https://doi.org/10.1007/s00441-0152212-x)

Yoon JW, Lee JS, Kim BM, Ahn J \& Yang KM 2014a Catechin-7-Oxyloside induces apoptosis via endoplasmic reticulum stress and mitochondrial dysfunction in human non-small cell lung carcinoma H1299 cells. Oncology Reports 31 314-320. (https://doi.org/10.3892/ or.2013.2840)

Yoon SB, Choi SA, Sim BW, Kim JS, Mun SE, Jeong PS, Yang HJ, Lee Y, Park YH, Song BS et al. 2014b Developmental competence of bovine early embryos depends on the coupled response between oxidative and endoplasmic reticulum stress. Biology of Reproduction 90 104. (https:// doi.org/10.1095/biolreprod.113.113480)

Yoshida H 2007 ER stress and diseases. FEBS Journal 274 630-658. (https:// doi.org/10.1111/j.1742-4658.2007.05639.x)

Yun SP, Yoon YM, Lee JH, Kook M, Han YS, Jung SK \& Lee SH 2018 Tauroursodeoxycholic acid protects against the effects of P-cresolinduced reactive oxygen species via the expression of cellular prion protein. International Journal of Molecular Sciences 19 352. (https://doi. org/10.3390/ijms19020352)

Yuzefovych LV, LeDoux SP, Wilson GL \& Rachek LI 2013 Mitochondrial DNA damage via augmented oxidative stress regulates endoplasmic reticulum stress and autophagy: crosstalk, links and signaling. PLOS ONE 8 e83349. (https://doi.org/10.1371/journal.pone.0083349)

Zhang JY, Diao YF, Oqani RK, Han RX \& Jin DI 2012 Effect of endoplasmic reticulum stress on porcine oocyte maturation and parthenogenetic embryonic development in vitro. Biology of Reproduction 86128. (https://doi.org/10.1095/biolreprod.111.095059)

Received 14 October 2019

First decision 7 November 2019

Revised manuscript received 7 January 2020

Accepted 16 January 2020 